\title{
Application of Ensemble Kalman Filter to Pedestrian Flow
}

\author{
Fumiya Togashi' ${ }^{1}$, Takashi Misaka ${ }^{2}$, Rainald Löhner $^{3}$, Shigeru Obayashi $^{4}$ \\ ${ }^{1}$ Applied Simulations Inc. \\ 10001 Chartwell Manor Cr., Potomac, MD 20854, USA \\ fumiya.togashi@gmail.com \\ ${ }^{2}$ Frontier Research Institute for Interdisciplinary Science, Tohoku University \\ 2-1-1 Katahira, Aoba-ku, Sendai 980-8577, Japan \\ misaka@edge.ifs.tohoku.ac.jp \\ ${ }^{3}$ George Mason University \\ 4400 University Dr., Fairfax, VA 22030, USA \\ rlohner@gmu.edu \\ ${ }^{4}$ Institute of Fluid Science, Tohoku University \\ 2-1-1 Katahira, Aoba-ku, Sendai 980-8577, Japan \\ obayashi@ifs.tohoku.ac.jp
}

\begin{abstract}
We adopted the Ensemble Kalman Filter (EnKF) methodology in our computational simulation code for pedestrian flows. The EnKF, which is a type of data assimilation methodology, has been developed in the field of weather forecast where the atmospheric condition varies hour by hour. The EnKF estimates the parameters or boundary/initial conditions in the numerical model based on the updated measured data. We considered the EnKF a promising tool for the simulation of pedestrian flows, which are notoriously difficult to predict. In this study, two scenarios were conducted to confirm the usefulness of the EnKF. The first case was unidirectional pedestrian flow in straight corridors, and the second case was Mataf scenario at the Kaaba in Mecca. Needless to say, the second scenario was very challenging because of the number of pilgrims and the degrees of freedom. In each scenario, we conducted the numerical simulation using the original parameter set and then applied the EnKF to improve the accuracy of the simulation.
\end{abstract}

Keywords: Computational Crowd Dynamics, Ensemble Kalman Filter, Data Assimilation, Pedestrian flow

\section{Introduction}

During the past decade, research on pedestrian and traffic flows has attracted a lot of attention $[1,2]$. With the aid of supercomputers, one can consider a large number of scenarios in a short period of time. However, human behavior has individual variability, so typical models end up having many empirical parameters. Generalization of these parameters, which determine the human behavior that is ultimately simulated, could be very challenging to determine as they vary depending on circumstances (age group, cultural characteristics, etc.). The aim of the present study is to determine these parameters in an efficient and automatic way from experiments. One promising candidate for such an automatic parameter estimation is the Ensemble Kalman Filter (EnKF) methodology [3, 4]. In this study, our objective is to confirm the usefulness of the EnKF in estimating the proper parameters based on the updated observation data (see [7] for a detailed description).

\section{Ensemble Kalman Filter (EnKF)}

EnKF is a type of sequential data assimilation methodology which has been developed from the Kalman Filter [5]. In the Kalman Filter, the model is integrated forward in time and, whenever the observation data become available, it is used to update the model prediction before the integration continues. The methodology is often used in the field of weather forecast. The detail of implementation can be found in many references $[3,4]$. 


\section{Application of EnKF and Result}

In this study, the experiment of unidirectional flow in straight corridors was utilized as the observation data. A total of 28 runs were performed in corridors with widths of $1.8 \mathrm{~m}, 2.4 \mathrm{~m}$, and $3.0 \mathrm{~m}$. The details of exit and entrance width in the experiment setting can be found in ref [6]. Figure 1 shows the snapshots from the experimental run. In the experiment, averaged pedestrian density, velocity in the corridors, and evacuation time (time until the last pedestrian reached the exit) were measured. Numerical simulations were conducted for all 28 runs. Figure 2 shows the blueprint of the numerical simulation and the computed result. The pedestrians are represented by the dots in the numerical simulation. The comparisons of the evacuating time between the experiment (green) and the computations (blue) are shown in Figure 3. The focus was on improving the input parameters for the worst case: run 28.

EnKF was applied to improve 8 empirical parameters, namely, desired pedestrian velocity, variability of velocity, relaxation time to achieve desired velocity, variability of relaxation time, variability of pedestrian radius, pushiness $(\mathrm{min} / \mathrm{max})$, and comfort zone. The measured pedestrian density and velocity were utilized as the observation data for EnKF. 20 parameter sets were prepared as the initial ensemble members. The total computational time was a few hours using a laptop, or about 7 minutes using 576 cores of the SGI ICE X system. The filtering processes took a few seconds.

After applying EnKF, the new parameter set was applied to all 28 cases. The evacuation time comparisons of all runs were shown in Fig. 3 as red dots. Remarkably, using the new parameters improved the computed evacuation times for all of the runs.
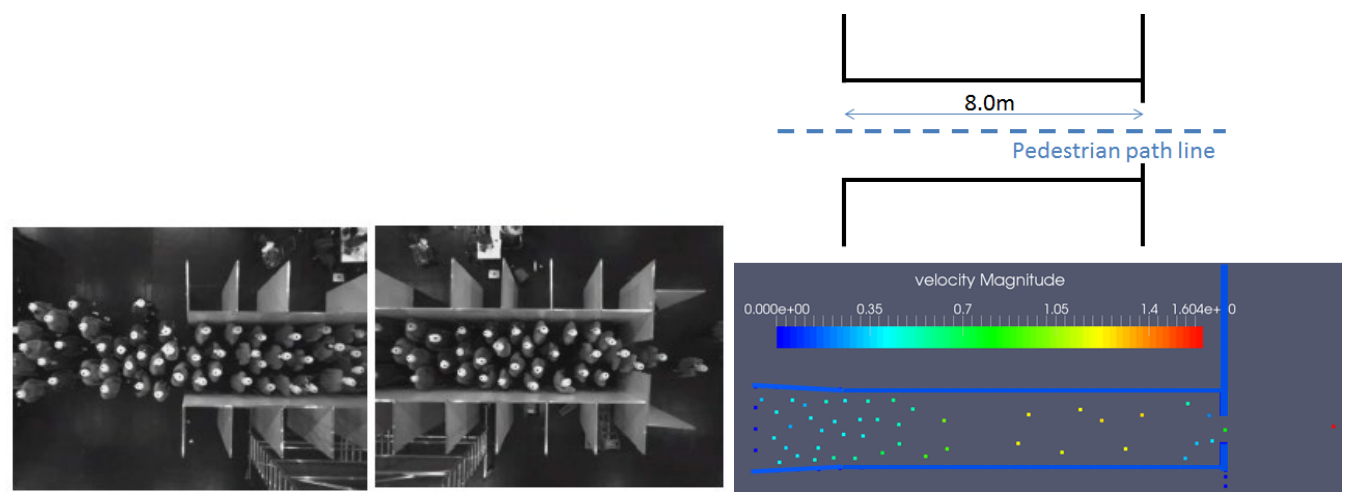

Fig. 1: Left: Snapshots from one run of the experiment. Fig. 2: The blueprint and snapshot of the numerical simulation

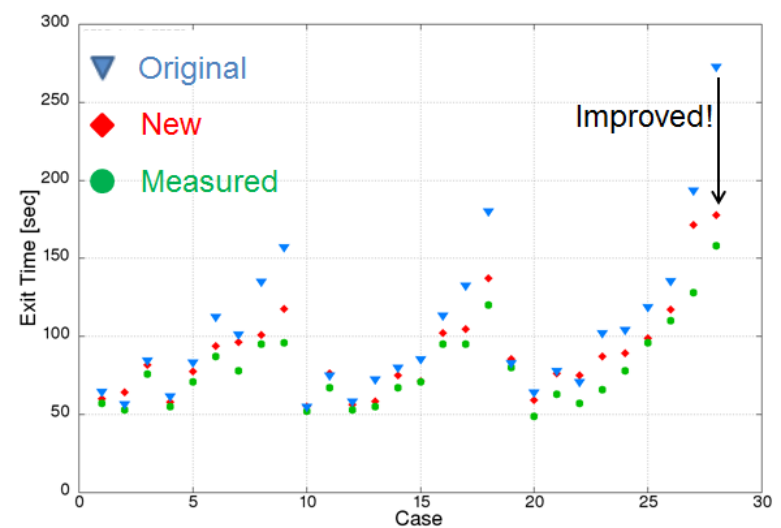

Fig. 3: Comparison of evacuating time of all 28 runs between the experiment (green) and the computed results using the original parameters (blue), and using the estimated parameters (red) 


\section{Mataf at the Kaaba in Mecca}

In the second case, the real observation data of pilgrims around the Kaaba was utilized to improve the accuracy of the numerical simulation. In addition to the 8 parameters which were handled in case 1 , two more parameters were added for improvement: the pilgrim's will force and tolerance to high densities. 32 parameter sets were prepared as the initial ensemble members. The total computational time was about 20 hours using 576 cores of the SGI ICE X system. Figure 7 shows the snapshot of the numerical simulation using the estimated parameter set by the EnKF and the comparison of the pilgrim density along the distance from the Kaaba for the observation, the computed result using the original parameter set, and the computed result using the estimated parameter set. The computed result using the estimated parameter sets by the EnKF shows an excellent agreement with the observation.
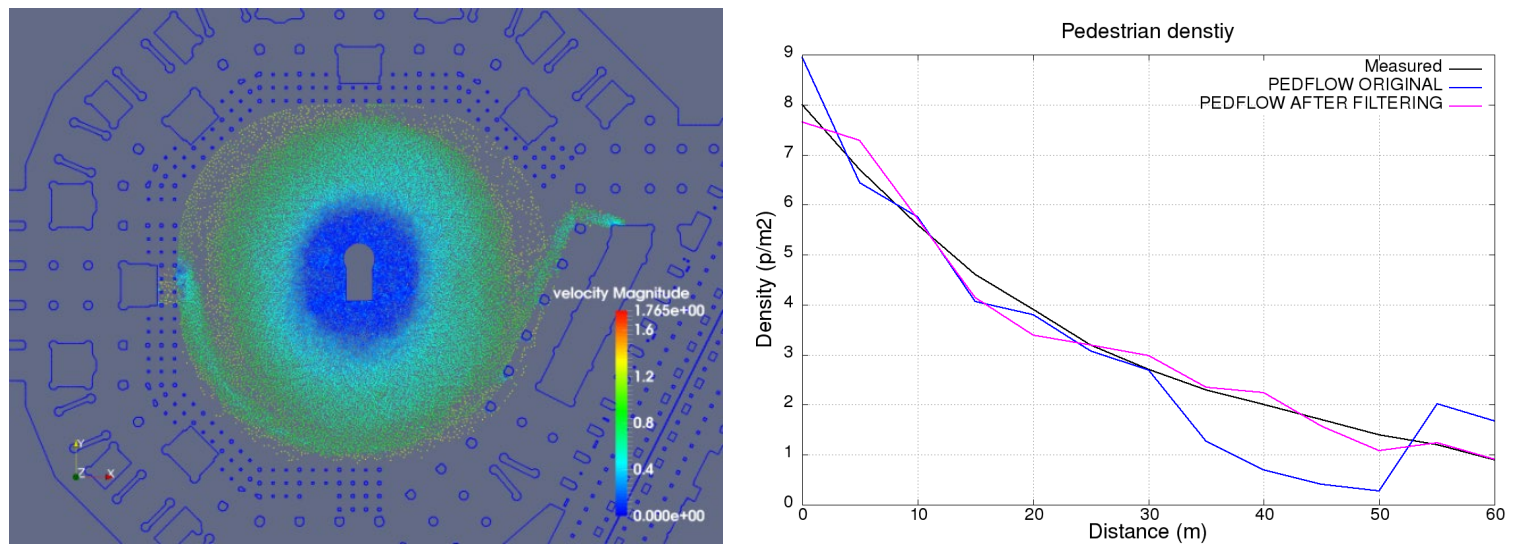

Fig. 6: (Left) Snapshot of the numerical simulation of pilgrims around the Kaaba using the new parameter set. (Right) Comparison of the pilgrim density along the distance from the Kaaba between the observation (black) and the computation using the original parameter set (blue), and the computation using the estimated parameter set

(magenta)

\section{Conclusion}

EnKF was applied to estimate the empirical parameters required by the pedestrian and crowd dynamics simulation code PEDFLOW [7]. The new runs using the estimated parameter set provided much closer results to the experimental data than the results using the original parameters.

The advantage of the EnKF is the capability of dynamically utilizing the observation data. Parameters, initial conditions, and boundary conditions are updated whenever the new observation data is available. Thus the accuracy of the numerical simulation keeps improving along the environmental conditions such as season, weather, time, temperature, etc.

The application of EnKF to pedestrian flow simulation will be a useful approach for future pedestrian flow simulations.

\section{References}

[1] The Conference on Pedestrian and Evacuation Dynamics, a biannual Conference; 2016 edition was in Hefei, China; see (W. Song, J. Ma and L. Fu eds.), University of Science and Technology Press,Hefei, China (2016).

[2] Helbing, Dirk, et al. "Simulation of pedestrian crowds in normal and evacuation situations." Pedestrian and evacuation dynamics 21.2, pp. 21-58, 2002

[3] E. Kalnay, “Atmospheric Modeling, Data Assimilation and Predictability,” New York: Cambridge University Press, 2003

[4] G. Evensen, "The ensemble Kalman filter: Theoretical formulation and practical implementation," Ocean Dynamics 53, pp. 343-367, 2003 
[5] Kalman, Rudolph Emil. "A new approach to linear filtering and prediction problems." Journal of basic Engineering 82.1, pp. 35-45, 1960

[6] J. Zhang, D. Britto, M.Charaibi, R., Löhner, E. Haug, B. Gawenat, "Quantitative validation of PEDFLOW for description of unidirectional pedestrian dynamics," Transportation Research

Procedia, 2, pp. 733-738, 2014

[7] F. Togashi, T. Misaka, R. Löhner, S. Obayashi. "Using Ensemble Kalman Filter to Determine Parameters for Computational Crowd Dynamics Simulations," Engineering Computations 35, 7, 2612-2628, 2018 